Design Characteristics of the $1.56 \mathrm{~m}$ Astrometric Telescope and its Usage in Astrometry

Wan Lai, Zhu Neng-hong, Wang Lan-juan, Yang Zheng-hua, Zheng Yi-jin Shanghai Observatory, Academia Sinica

The $1.56 \mathrm{~m}$ astrometric telescope (Fig. 1) is designed mainly for determining trigonometric parallaxes of faint stars.

In order to keep the design as simple as possible, the telescope has only a Ritchey-Chretien focus and will accomodate plates $200 \times 200 \mathrm{~mm}$. We also plan to design several pieces of auxiliary equipment such as a medium dispersion grating spectrograph, a photometer, a reducing camera, and an electrographic camera, all of which are in the second stage of engineering. The whole project consists of four parts.

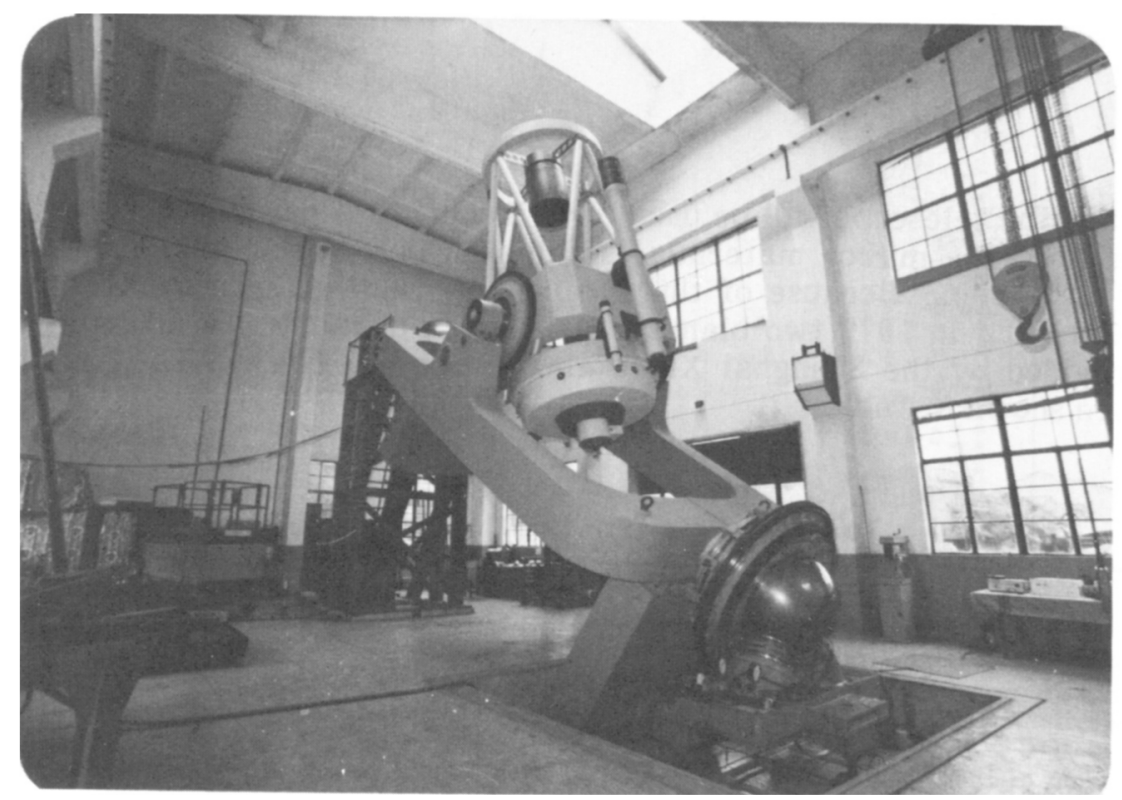

Figure $1 \quad 1.56 \mathrm{~m}$ astrometric telescope 275

H. K. Eichhorn and R. J. Leacock (eds.), Astrometric Techniques, 275-287.

(C) 1986 by the IAU. 
I. Optical System

The design of the optical system is based on the requirement that it should reach magnitude of $\mathbf{1 7 . 5}$ in 15 minutes of direct photography.

(1) Main optical system (Fig. 2)

The main optical system of the telescope is an Ritchey-Chretien (RC) system. It consists of a concave hyperbolic primary of $1560 \mathrm{~mm}$ diameter and a convex hyperbolic secondary of $530 \mathrm{~mm}$ diameter. The focal ratio if $F / 10$, the equivalent focal length of the main optical system is $15600 \mathrm{~mm}$ and the plate scale is therefore $13: 22 / \mathrm{mm}$. Table 1 shows some of the principal parameters of the primary and secondary mirrors.

Table 1 Main parameters of the primary and secondary

\begin{tabular}{|c|c|c|}
\hline & primary mirror & secondary mirror \\
\hline radius of curvature & $\mathrm{R}_{1} \quad 10400 \mathrm{~mm}$ & $\mathrm{R}_{2} \quad 4543.5 \mathrm{~mm}$ \\
\hline figure & concave & convex \\
\hline focal length & $5200 \mathrm{~mm}$ & $f_{2} \quad 2271.75 \mathrm{~mm}$ \\
\hline eccentricity & $e_{1}^{2} \quad 1.0919572$ & $e_{2}^{2} \quad 5.0663066$ \\
\hline aspherical degree & $12 \mu \mathrm{m}$ & $8 \mu \mathrm{m}$ \\
\hline
\end{tabular}

Since the telescope will be used for trigonometric parallax observations, it is essential that the mirror materials used have the lowest possible coefficient of thermal expansion. Because of this the primary and secondary mirrors are made of the Cer-Vit. In 1979 two blanks, $1600 \mathrm{~mm}$ in diameter and $270 \mathrm{~mm}$ thickness, were provided by the Shanghai Xinhu glass factory. The qualities of these two blanks are shown in Table 2.

Table 2 Qualities of the mirror blankes

\begin{tabular}{l|c|c}
\hline & $N_{0} 1$ & $\lambda_{2}$ \\
\hline maximum of the edge stress & $7.7 \mu \mathrm{m} / \mathrm{cm}$ & $7.1 \mu \mathrm{m} / \mathrm{cm}$ \\
\hline average diameter of tne bubble & $0.21 \mathrm{~mm}$ & $0.16 \mathrm{~mm}$ \\
\hline coefficient of thermal expansion & $2.0 \times 10^{-9}$ & $-0.28 \times 10^{-7}$ \\
\hline number of bubbles per litre & 260 & 390 \\
\hline
\end{tabular}




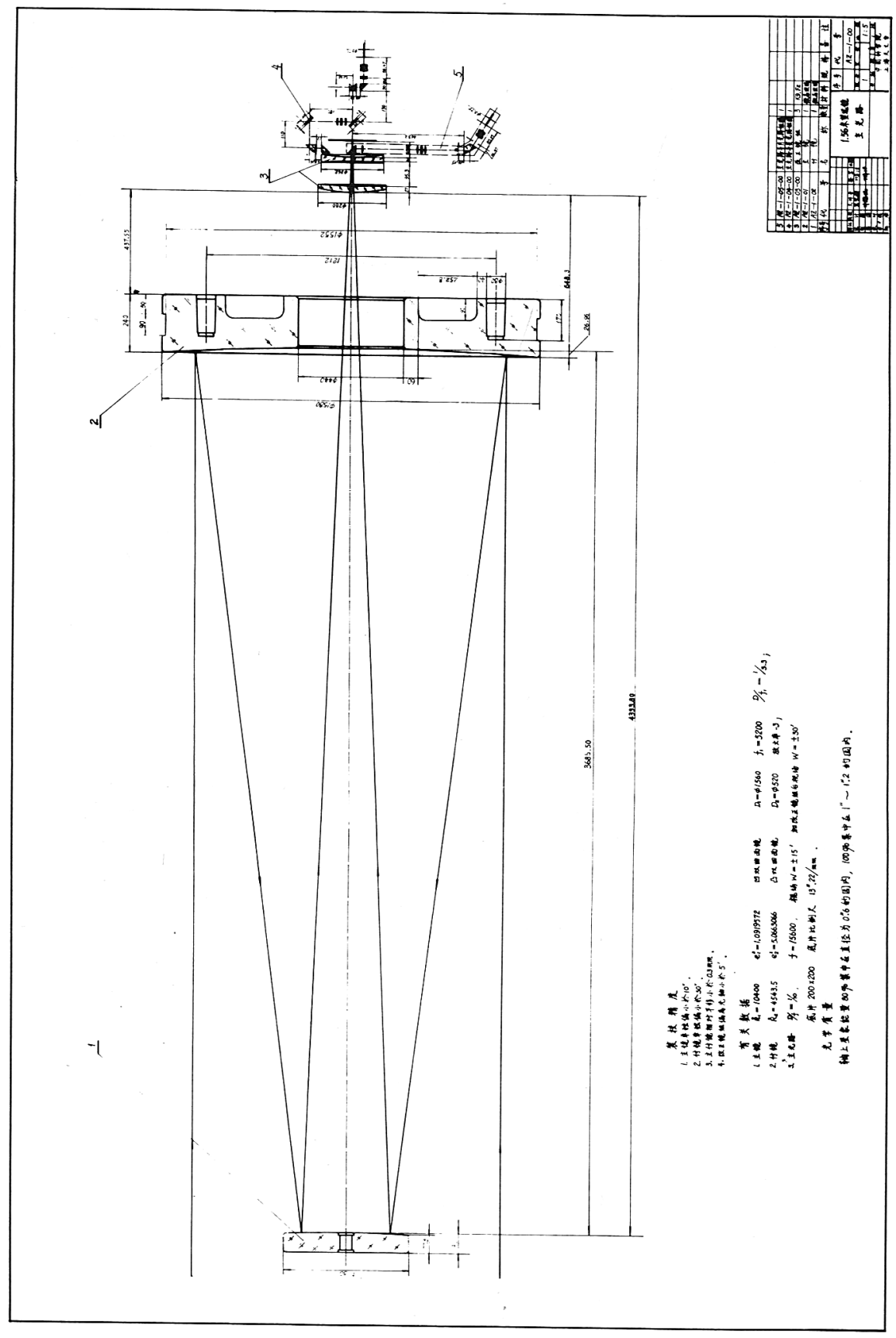

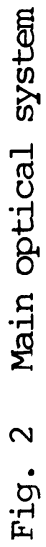


There were two reasons to choose a RC system. One is that the length of the telescope can be reduced considerably, the other, that the RC system can eliminate spherical aberration and coma simultaneously. Meanwhile we have paid a great deal of attention the limiting the effect of misalignment of the RC system which can cause coma.

The coma free field of the main optical system has a radius of $15^{\prime}$, sufficient for trigonometric parallax work. The field can be extended to a radius of $30^{\prime}$ for purposes other than high precision astrometric work by adding a field corrector. Optical calculation show that the maximum diameter of an image disc is less than $1^{\prime \prime}$ in a field of $10^{\prime}$ The maximum of monochromatic light speckle is 0.073 in the wavelength range of $4047 \AA$ to $6563 \AA$. Transverse chromatic aberration reaches a maximum of 0 !17. We also calculated the spot diagram in the wavelength range of $3650 \AA$ to $10140 \AA$. The maximum speckle of monochromatic light is 0 "148 and the maximum of transverse chromatic aberration at $10140 \AA$ is $0 ! 541$.

(2) The optical systems of the guiding telescope and the finder telescope.

Two guiding telescopes and two finders are attached to the two sides of the centerpiece of the tube. In this way, observations can be made no matter how the telescope is pointed.

Both the guiding telescopes and the finding telescopes are refractors. The clear aperture of the guiding telescope is $220 \mathrm{~mm}$ and its focal length is $2860 \mathrm{~mm}$. The field of view has a radius of $1: 5$.

(3) The off-set guiding system (Fig. 3)

In order to guide faint stars more accurately and to avoid a guiding error resulting from the flexure of the tube we have designed an off-set guiding system at the RC focus. This system must reach a limiting magnitude of 12 or 13 . The range of the off-set guiding area is an annulus of about 6 arcminutes at the edge of the field of the main optical system. The visual field seen in the microscope eyepiece measures $26 \times 20 \mathrm{~mm}$. The guider pick-up prism can rotate 270: around the optical axis in the annulus which is $26 \mathrm{~mm}$ wide. 


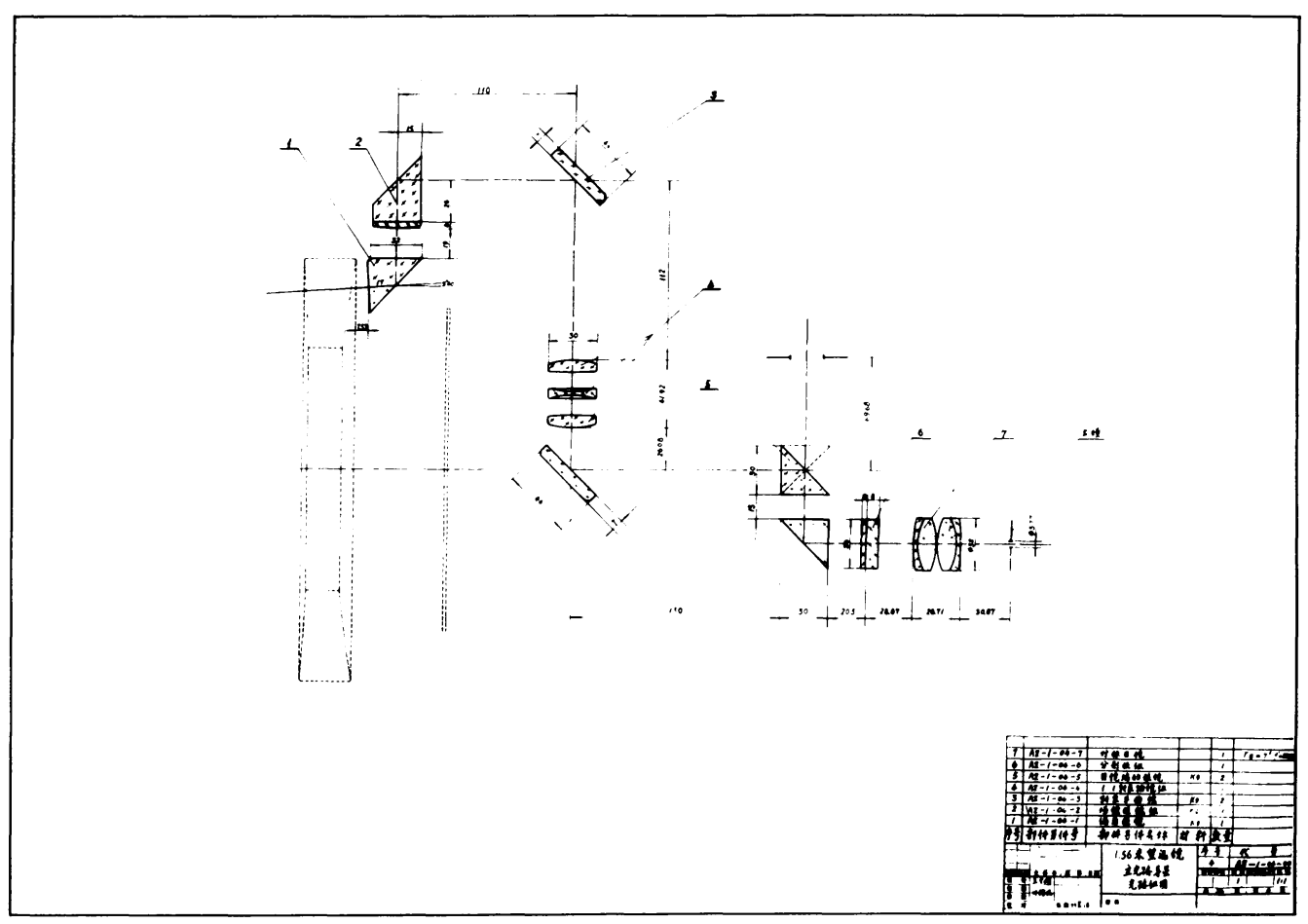

Fig. 3. Off-set guiding system

II. Mechanical Assembly

(1) Mounting

In order to be able to reach the polar region, we are mounting the telescope in a fork-yoke frame. This combination of fork and yoke is a symmetrical tubemounting with the characteristic of "equal flexure" and can also overcome the flexure problem of the polar shaft of a fork mounting. The fork-yoke mounting is formed by welding two short, strong fork arms to the top surface of the yoke frame. Two bearing supports for declination are screwed on to the upper plane of the short arms. The tube will thus not be covered by the northern pier when it is pointed north. This mounting increases the rigidity and strength of the yoke frame.

The northern and southern bearings of the frame are hydrostatic oil pads. They are hemispherical in shape and $1 \mathrm{~m}$ in diameter. Both azimuth and altitude of the polar axis can be aligned at the southern pier. The alignment range is $30^{\prime}$. 
(2) Tube

The tube of the telescope is a Serrurier type truss and consists of the primary mirror cell, the secondary mirror cell, the two mirror caps, the sky baffle, the multiple exposure camera, etc.

Fig. 4 is a schematic diagram of the primary mirror cell. The primary mirror is supported both axially and radially by eighteen level-counterweight mechanical systems, which are mounted in sockets in the back of the mirror.

Three of the axial support levers are micrometer-controlled for collimation. The principle for arranging the mirror supports is that each support system carry one eighteenth of the weight of the mirror (Fig. 5).

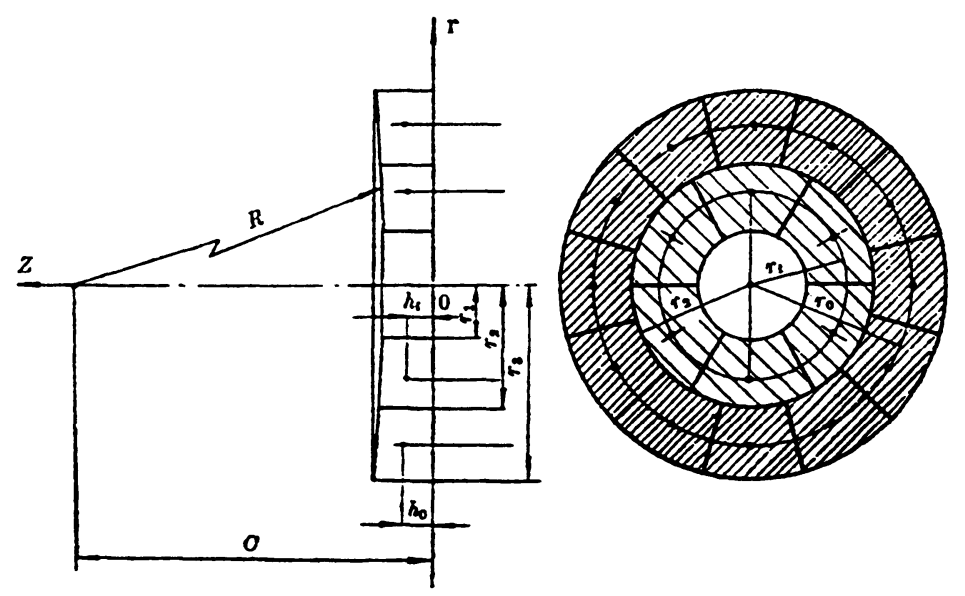

Fig. 5. The distribution of mirror supports

The circle with radius $r$ divides the mirror into two annului. The inner annulus is divided into six equal sectors, while the outer annulus is divided into twelve equal sectors. So the whole mirror is divided into eighteen sectors, each of which having the same mass.

The deformation of the primary mirror under its own weight has been calculated. The maximum deformation is $26 \mathrm{~m} \mu$, or about $\lambda / 21$, and thus satisfies the Raleigh criterion.

The radial position of the primary mirror is provided by four spherical screws which are attached to the central defining tube (Fig. 6). The defining tube, is also made of Cer-Vit, and is located in the central hole of the mirror. Its lower part has the form of a cone. The defining tube and mirror cell are connected 


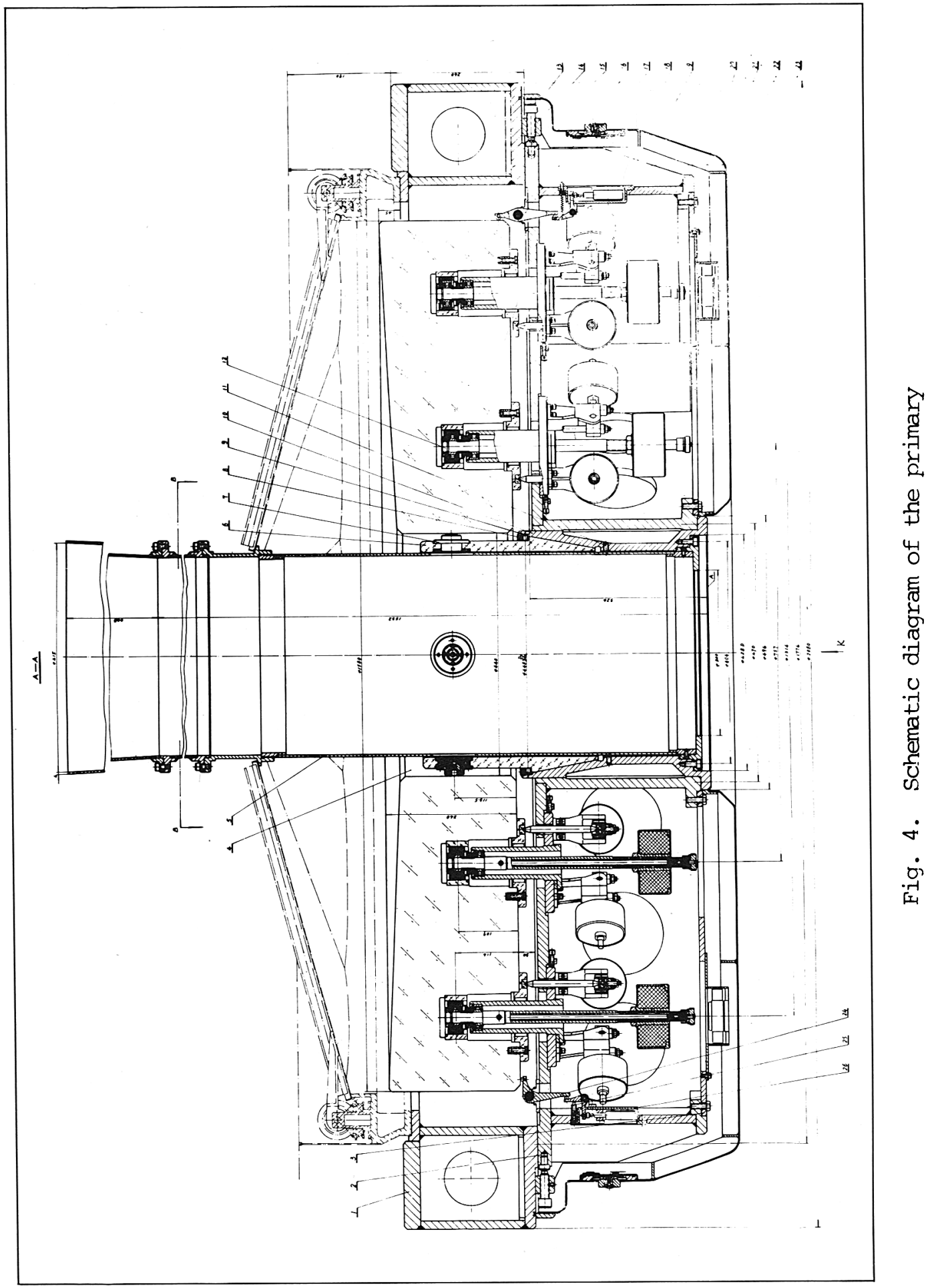


tightly by using a ring spring at the taper of the cone. This device solves the problem of the thermal effects of the thermal expansion between the cell and the mirror itself. Similarly, the thermal effect between the defining tube and the mirror cell is eliminated.

The secondary mirror is supported axially by atmospheric pressure because the cell is slightly evacuated behind the mirror. The edge support of the secondary mirror is provided by a mercury tube. A counterweight pressure changes the vacuum in the secondary mirror cell to generate the different support forces when the tube telescope is pointed to different parts of the sky.

A multiple exposure camera was designed to conserve observation time, which is important for a parallax program. The camera holds four plates which can be exchanged automatically one by one (Fig. 6) and the exposure time can be controlled.

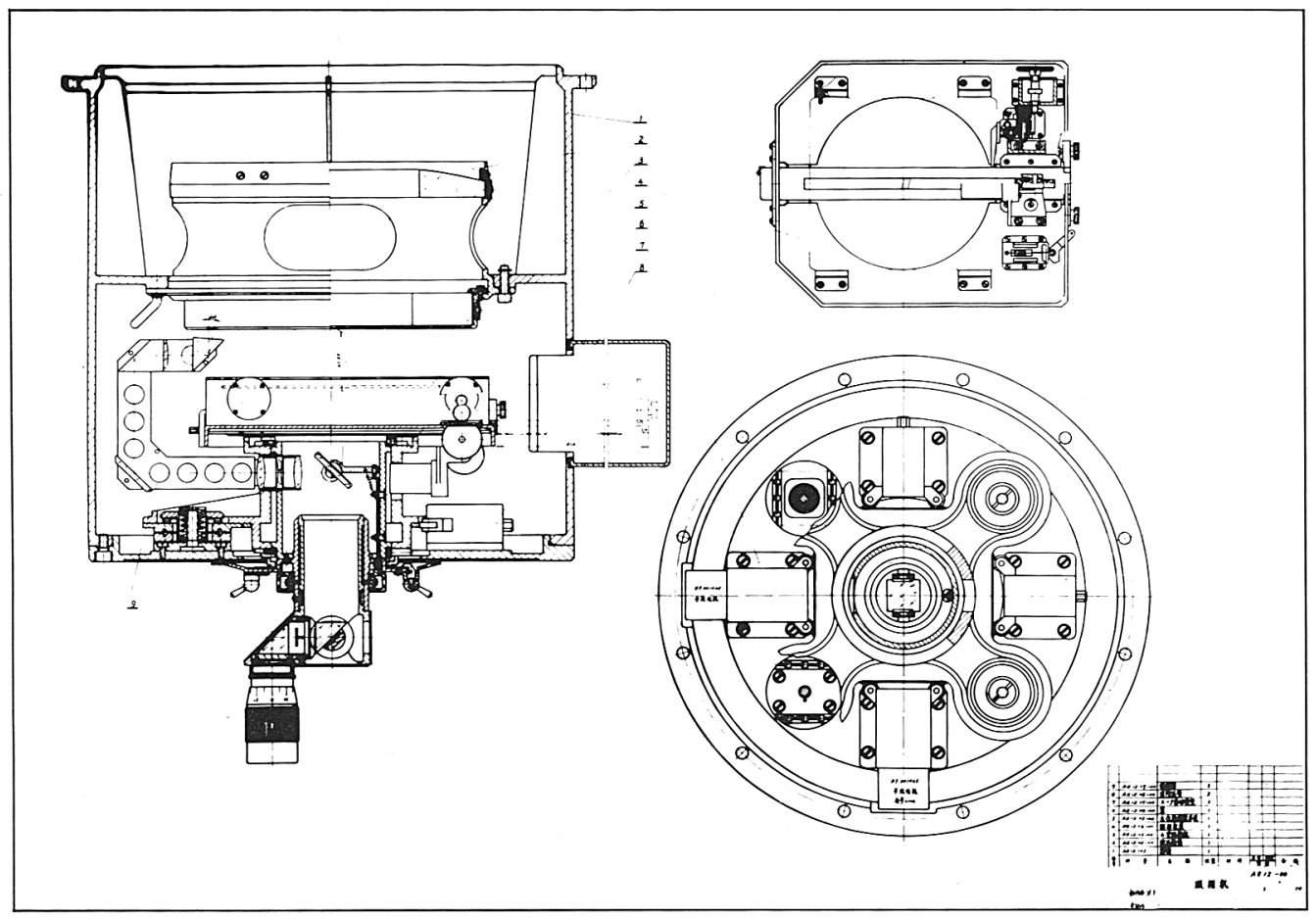

Fig. 6. Camera 
(3) The automatic focusing device (Fig. 7)

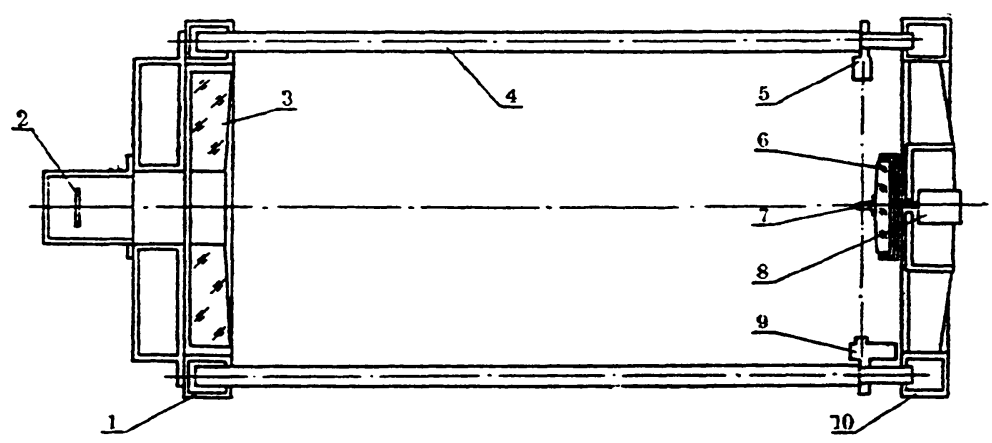

1. primary mirror cell

2. plate

3. primary mirror

4. Cer-Vit rod

5. light source and slit

6. secondary mirror

7. thin lens

8. focusing motor

9. microscope objective and C. C. D

10. up ring

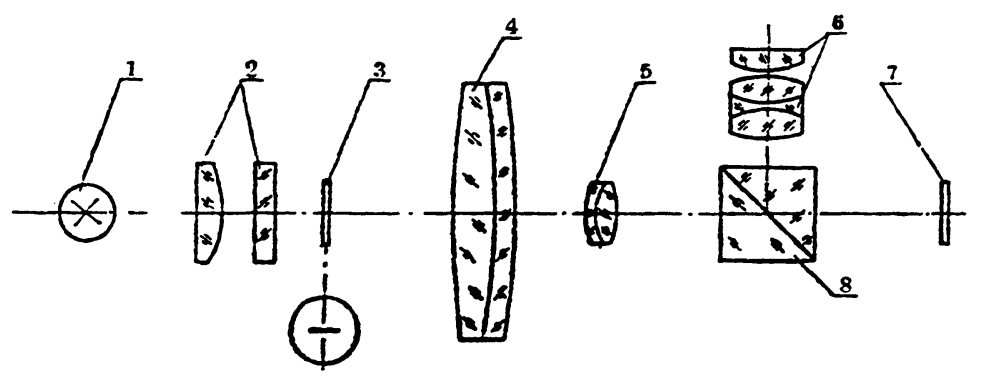

Fig. 7. Schematic digram of the Auto-focusing and its optical system
1. light source
2. condensing lens
3. slit
4. thin lens

5. microscope objective

6. eyepiece

7. C. C. D

8. cubic prism

Because primary and secondary mirrors are made of Cer-Vit, the optical configuration is not very sensitive to temperature changes during observation. Since the tube is made of steel, any change in the separation between primary and secondary mirror is a serious problem. Such a change will cause the focus to drift. We have designed a device for automatic focusing.

Two Cer-Vit rods keep the distance change between the primary and secondary mirrors within the permitted tolerance. These run from the back surface of the primary to the top ring of the Serrurier truss. A slit mounted on the upper end of one rod is illuminated by a light source through a condensing lens.

A thin lens is fixed at the center of the secondary mirror. A microscope objective mounted on the upper end of another Cer-Vit rod and a CCD element is 
located in the focal plane of the microscope objective. The image of the slit is formed at the objective space of the microscope. After alignment of the main optical system, the position of CCD is adjusted such that there is no output. When the temperature changes at night, the length of the Cer-Vit rod will not change but the tube will expand. The thin lens moves with the secondary mirror. Hence the image of the slit will move in the plane of the CCD. This motion generates output through the CCD. This signal is then amplified and drives a step motor which returns the secondary mirror to the optimum position.

(4) Telescope drive

The total weight rotated about the polar axis is approximately 32 tons. Because hydrostatic oil pads are used for the bearings of the polar axis it is possible to use a single worm wheel for both slewing and tracking in right ascension. The right ascension worm gear is $1875 \mathrm{~mm}$ in diameter and has 625 teeth. The declination drive is similar to the right ascension drive. Its worm gear is $1500 \mathrm{~mm}$ in diameter amd has 500 teeth. Both for right ascension and declination a DC motor is used to generate the different rates for slewing, tracking, setting and guiding. The slew rate is 90: per minute, the set rate is $1: /$ minute and the guide rate is 1 ' per minute.

The tracking rate is variable and can differ from the sidereal rate up to $\pm 5 \%$. The declination drive rates are the same as the right ascension drive except for the tracking rate, which is variable from 0 to $20^{\prime \prime}$ per minute. There is an overload safety device in the drive system to prevent any accidents.

III. Control System

(1) Drive control of the telescope

Two high angular speed DC motors are used to drive both the polar and the declination shafts. The motor and several resolvers consistitute a digital speed servo system to realize the slew, set, guide and various sidereal rates. The sidereal speed can be finely adjusted with a switch on the console. There are another four resolvers used for measuring the angular positions of the polar axis and the declincation axis to set the telescope automatically. The block diagram of the drive control system is shown in Fig. 8. 


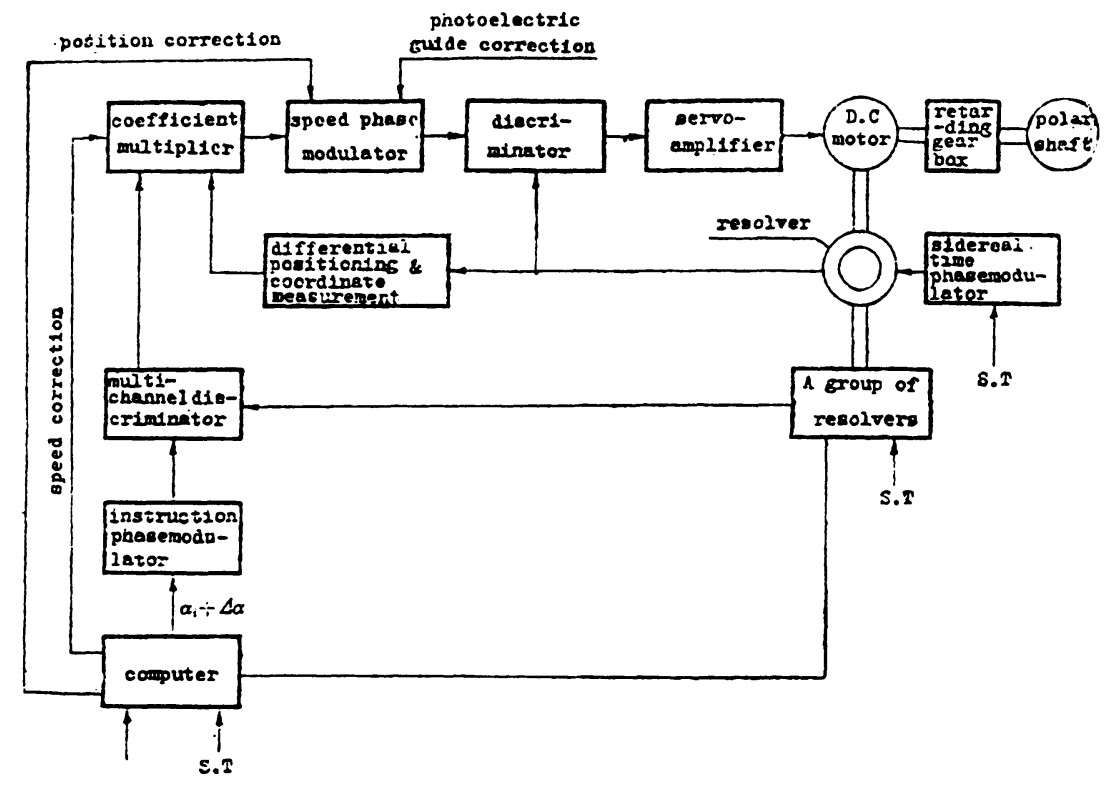

Fig. \& Block diagram of the polar axis driving system

(2) Photoelectric autoguide system

The above mentioned off-set guiding at the back of the primary mirror cell, is equippped with a reflecting prism. By reversing the prism by 90:, the image of the star which should be guided will be folded to a semicircular knife disc located in front of the photomultiplier. This semicircular knife dise is rotated at 396 rpm. If the center of the star image coincides with the semicircular disc the photomultiplier has no output. Otherwise a guide error will be produced.

A small guiding error can be corrected by shifting the plateholder, but a large guide error must be corrected by moving the telescope.

For reaching fainter guide stars in order to improve the accuracy of this autoguide system, a single photon-electron counting technique has been used. Its block diagram is given in Fig. 9. 


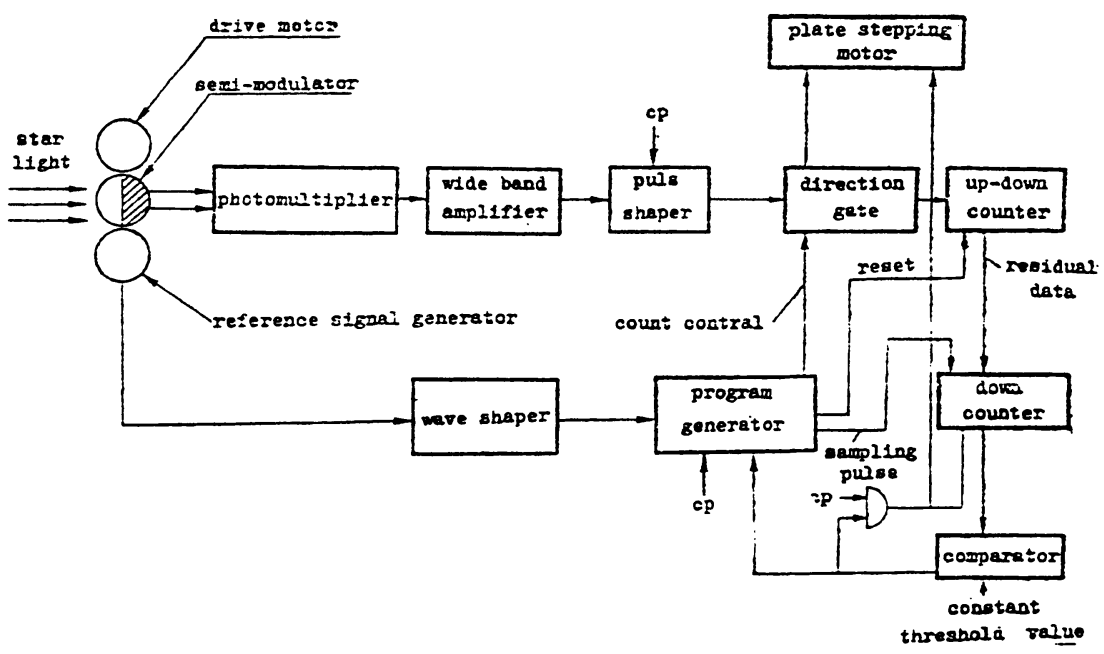

Fig. 9 Block scheme of photoelectric guidance system

(3) Dome and windscreen servo system

A special digital computer transforms the position of the telescope altazimuth coordinates. Thus calculating the azimuth of the dome window and the zenith distance of the windscreen. The actual position of the dome and the windscreen are given by the encoders. By means of a subtractor we can obtain the difference between the computer output and the encoder and thus control the DC motor of the dome and the windscreen.

\section{Observatory Building}

The building will be constructed on the west slope of Zo-Se in the western suburb of Shanghai. The inner diameter of the dome is about $17 \mathrm{~m}$. The center of the dome is elevated about $21.7 \mathrm{~m}$ above the ground floor of the building. The total height from the ground floor to the top of the dome is about $30 \mathrm{~m}$.

An aluminizing room, will be located on the ground floor of the building. The optical laboratory and the electric control room will be located on the first floor. The dome itself will be located on the second floor.

As this is written, the primary mirror of the telescope is undergoing testing, and the principle components of the telescope have been manufactured and moved into a special workshop and assembly room in our observatory. Construction of the dome building for the telescope will begin soon. The telescope will be installed at Zo-Se at the end of 1985 or the beginning of 1986 if everything goes smoothly. 
The first astrometric reflector in the world is the $1.55 \mathrm{~m}$ reflector of the United States Naval Observatory. Our reflector, when finished, will be the first telescope of this kind in Asia.

The principal goals of our $1.56 \mathrm{~m}$ astrometric reflector are:

(1) The determination of trigonometric parallaxes for some nearby faint stars, such as red dwarfs, white dwarfs and subdwarfs, and for some nearby clusters such as the Hyades. These should yield useful information for the investigation of the cosmic distance scale.

In addition, selected nearby cool carbon stars and quadruple systems will be put on our parallaxes program.

We are planning to determine parallaxes of some 30 stars per year with magnitudes down to $17 \mathrm{~m}$ and even fainter magnitudes with hypersensitized plates. Precisions are expected to correspond to standard errors of about 0:004.

(2) Determination of precise optical positions of some radio sources for future calibration of radio observations and of positions of planets and natural satellites.

Discussion:

HARRINGTON I would suggest you include a few quasars on your observing program. They make wonderful calibrators, since you know a priori exactly what their parallaxes are. 